http://www.pakjas.com.pk

\title{
Incidence of fusarium wilt in major tomato growing areas of Punjab
}

\author{
Hira Nawaz ${ }^{1}$, Muhammad Amjad Ali ${ }^{1}$, Rana Muhammad Atif ${ }^{2}, \mathrm{Ahmad} \mathrm{Nawaz}^{3}$ and \\ Amjad Abbas ${ }^{1, *}$ \\ ${ }^{1}$ Department of Plant Pathology, University of Agriculture, Faisalabad, Pakistan; ${ }^{2}$ Department of Plant Breeding and Genetics, \\ University of Agriculture, Faisalabad, Pakistan; ${ }^{3}$ Department of Entomology, University of Agriculture, Faisalabad, Pakistan \\ *Corresponding author's e-mail: amjad.abbas@uaf.edu.pk
}

\begin{abstract}
Tomato crop is affected with several diseases that lead to decreased yield every year. Among those diseases, tomato wilt is the most significant disease caused by soil borne pathogens i.e. Fusarium solani, Fusarium oxysporum, and Verticillium spp. In the present study, survey was conducted in tomato growing areas of Punjab. Pathogens associated with plants were Fusarium oxysporum, Alternaria solani, parasitic nematodes, Pythium, Verticillium dahliae and mosaic virus. The survey was conducted at three different stages of plant development and disease incidence and severity were correlated with environmental factors. This survey was conducted for consecutive two years (2017-18:2018-19). It was shown that fusarium wilt can infect all growing stages (nursery, flowering and fruiting) of tomato plant. Moreover, high temperature and high humidity favored disease prevalence. Huge damage was witnessed due to high temperature and relative humidity up to a certain limit. Furthermore, it was also observed that alternaria blight, verticillium wilt, nematodes and mosaic virus also attacked at different growth stages to different extents along with fusarium wilt. Plants were more vulnerable at fruiting stage to all diseases. The present study will be helpful for devising a proper management strategy for fusarium wilt disease in tomato crop.
\end{abstract}

Keywords: Tomato wilt, Fusarium oxysporum, seedling rot, epidemiology.

\section{INTRODUCTION}

Tomatoes (Solanum lycopersicum L.) are edible red berries belong to family Solanaceae. It was originated from west of South America and considered as second most imperative vegetable crop. Tomato is $2^{\text {nd }}$ most important crop and is being grown all over the world. (Srivastava et al., 2010). It contains many minerals and antioxidants (Nahar and Ullah, 2012). The Food \& Agriculture Organization ranks it at sixth position among the world's most common vegetables in total annual production. Tomato is the leading vegetable and is mainly grown in countries like China, India, USA, Turkey, Egypt, Iran, Italy, Brazil and Spain. These countries produce more than 74 percent of its annual world production, generates an annual total of 159 million tons of fresh tomato, of which about one quarter is cultivated for processing purpose. There is a major economic importance of tomato in Pakistan. Tomato consumption and demand are increasingly growing because of a population growth. Second important reason of tomato consumption is its availability during all the seasons and comparatively lower price. It is consumed in fresh from as salad or cooked in household meals in various dishes. Processed forms like ketchup and sauces of tomato are very common. It is also available throughout the year in various locations around the world due to its seasonal flexible production (Chohan and Ahmad, 2008). During 2009-2010, in Pakistan the area under tomato cultivation was 50 thousand hectares with a production of 9832 tons. Exports of tomato in the raw or processed form generated revenue of 77 million rupees. Europe and America are leading in tomato germplasm exploitation which resulted into various famous cultivars and such germplasm is considered as the base of commercially grown varieties in Pakistan (Nonari et al., 2015).

Tomato is susceptible to many plant pathogens. In tomato production, numerous species of Fusarium cause extensive losses (Wang et al., 2011). The pathogen survives in the soil and infects plants by the roots and the crown. Different Fusarium species are related to tomato varieties, for example $F$. equiseti, F. verticillioides, and F. oxysporum (Rozlinah et al., 2010). Under field conditions tomato yield is severely affected by wilt disease caused by Fusarium oxysporum $\mathrm{f}$. sp. lycopersici (Medeiros et al., 2009; Agrios, 2005). Fol prevails

Nawaz, H., M.A. Ali, R.M. Atif, A. Nawaz and A. Abbas. 2021. Incidence of fusarium wilt in major tomato growing areas of Punjab. Pak. J. Agri. Sci. 58: $1205-1213$.

[Received 5 Sep 2020; Accepted 29 Jun 2021; Published (online) 21 Sep 2021] 
in all tomato growing regions of the world. Fusarium wilt is a soil borne disease in nature and pathogen survives in the soil as chlamydospores. Fusarium is capable to survive in soil and retains its pathogenicity up to six years under suitable conditions. On germination of tomato seeds, Fol invades tiny seedlings of tomato through outer cell layer of roots and continues to grow.

Rise in average soil temperature favors fusarium in its pathogenicity on young seedlings as well as mature plants (Ignjatov et al., 2012). As the fusarium grows in the vascular tissue, transportation of water and salts to photosystem is halted which is observed as peculiar symptom of wilting or chlorosis in young seedlings (Zvirin et al., 2010). At any growth stage of the plant, Fusarium can infect both in the roots and crown. Some species, including Fusarium oxysporum, infect vascular bundles that cause infected plants, exhibit stress-related early wilt syndromes (Adisa et al., 2018). In acidic sandy soils, fusarium wilt is more natural. The wilt pathogen can live up to ten years in infested soil. Higher soil temperatures like $34^{\circ} \mathrm{C}$ favors wilt disease while cooler soils with $17-20^{\circ} \mathrm{C}$ delay the occurrence of wilt (Di Pietro et al., 2003). Previously, the population of Fusarium species associated with tomato wilt has not been studied in detail.

The present study emphasizes thirteen different locations of Punjab Province. Sampling was done for two consecutive years. Severity of Tomato wilt disease was correlated with environmental factors to find out the optimum conditions conducive for disease epidemic. It is not clear, whether tomato-infecting Fusarium pathogen, currently prevails as single specie or it is a complex of multiple species in this area and whether these are monomorphic or polymorphic and which of them is more serious threat to tomato crop.

\section{MATERIALS AND METHODS}

Surveys and Sample Collection: Five districts (tehsils) of Punjab Province were selected for diseased sample collection. Typical symptoms were examined during sampling were wilted leaves from the base of plant and browning of stem from the middle. Five locations were selected from each major area and five sites per location were finalized for sampling. Surveys were done in X-shaped path and samples were selected at three growth stages like nursery, flowering and fruiting stages for two consecutive years. Diseased samples were brought to lab, debris and soil particle removed by washing with tap water. Samples were dried out by folding of sterile tissue paper and packed into zipper bags at $4^{\circ} \mathrm{C}$ (Akbar et al., 2018). Disease incidence and severity were correlated with environmental factor i.e. maximum temperature, minimum temperature, wind speed and relative humidity.

Isolation and Purification: Samples were washed properly and cut into smaller pieces, washed with $70 \%$ ethanol and placed on artificial media. Plates were placed in incubator at $27 \pm 2^{\circ} \mathrm{C}$ for 3 days. After 3 days fungal growth was picked with needle and placed on PDA media plate. After 7 days fungal mycelia and spores were identified under microscope. Nematodes were also isolated by Berrman funnel method. Plants roots were wrapped in tissue paper and placed on sieve and placed on funnel containing water cover it with lid and

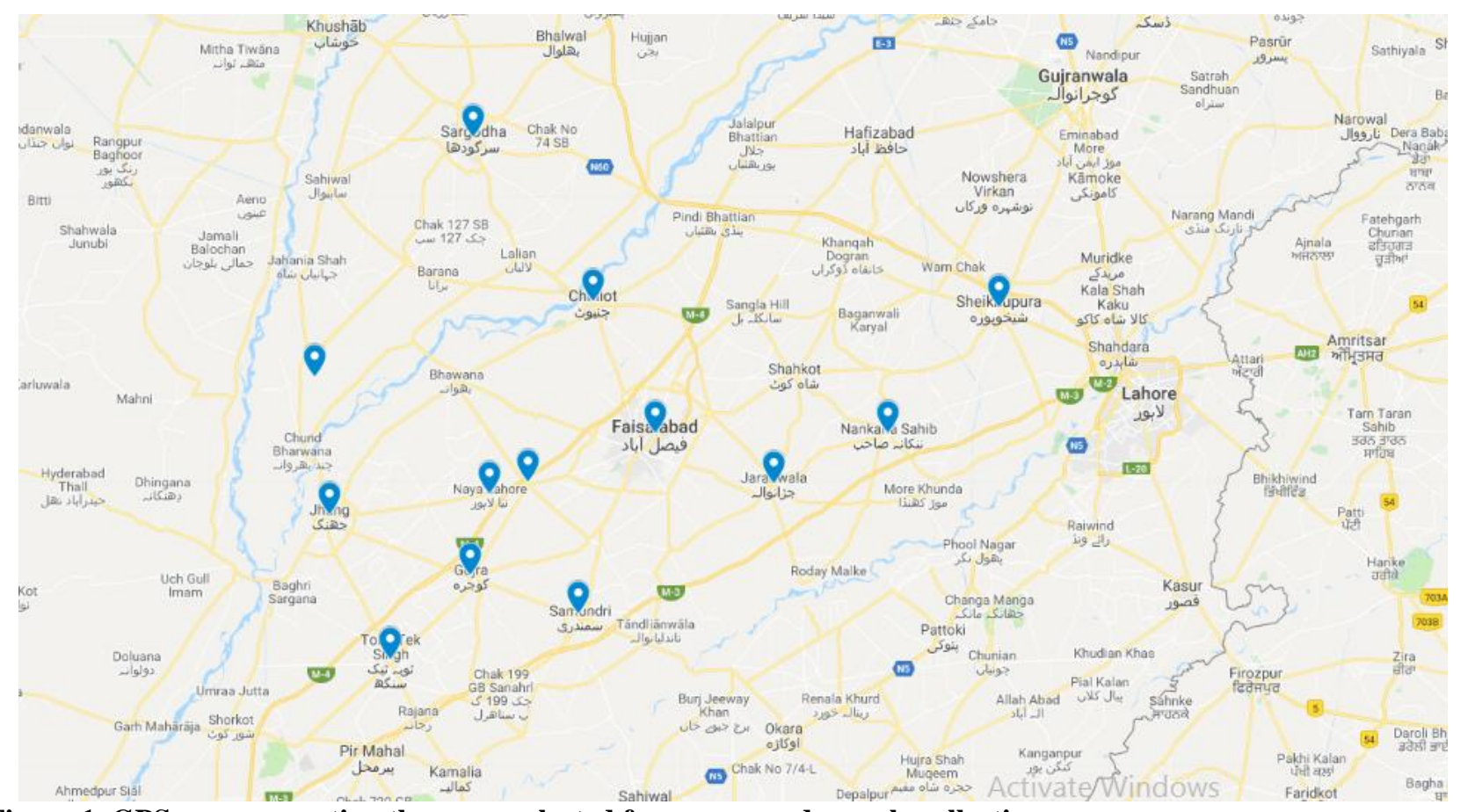

Figure 1. GPS map presenting the areas selected for survey and sample collection 
hang apparatus for 24 hours. Water near the stopper collected in petri plate and observed under stereomicroscope.

Tomato samples showing viral symptoms were grinded with pestle and mortar using extraction buffer and strip ELISA were performed for confirmation of viral attack.

Data analysis: Two-year data were analyzed by using statistical tools and formulas given below

Disease Severity $(\%)=\underline{\text { Infected plant area }} \times 100$

Total plant area

Incidence $(\%)=$ Total no. of diseased plant $\times 100$

Total no. of examined plants

The prevalence of wilt in each district was calculated as follows:

Prevalence $(\%)=\underline{\text { No. of field affected with } \mathrm{Fol}} \times 100$

Total no. of fields examined

The data were analyzed by using SAS 9.0 software and means were compared using LSD at $5 \%$ probability level. The regression analysis was done using Microsoft Excel version 2016.

\section{RESULTS AND DISCUSSION}

Survey was done for two consecutive years (2017-18; 201819) and similar results were recorded for both years. It was found that six different types of pathogens were prevailing in these thirteen areas which were under consideration, but prevalence varied with area and growth stage. Symptoms of different pathogens, growth on the media plates and spores are illustrated in Fig. 2.

In Jhang disease incidence of Fusarium wilt, damping off, Verticillium wilt, nematodes, and mosaic virus were found with different intensity. At nursery stage incidence of Fusarium wilt was (26\% and $33.34 \%$ at two places of Mandi Shah Jewana), Alternaria (3.4\%), damping off (11.3\%), nematodes (17.67\%), Verticillium wilt (15.67\%) and mosaic virus $(1.23 \%)$ recorded. Similarly, at flowering stage Fusarium wilt was (56\% and $49.67 \%$ in Mandi Shah Jewana), Alternaria $(0.5 \%)$, damping off $(0.5 \%)$, nematodes $(0.6 \%)$, Verticillium wilt $(33 \%)$ and mosaic virus $(0.5 \%)$. At fruiting stage Fusarium wilt incidence was recoded (62\% and $60.4 \%$ in Mandi Shah Jewana), Alternaria (34\%), damping off $(0.5 \%)$, nematodes $(31 \%)$, Verticillium wilt $(43 \%)$ and mosaic virus $(43 \%)$.

In Faisalabad, diseases associated with tomato crop at nursery, flowering and fruiting stage were monitored as shown in Fig. 1. At nursery stage Fusarium wilt incidence was $25.67 \%$ in Sumandri, $26 \%$ in Jaranwala, $28.6 \%$ in Pansera, $25.6 \%$ and 26.8 at two locations of Manawala. While incidence of Alternaria (3.4\%), damping off $(17.67 \%)$, nematodes $(1.34 \%)$, Verticillium wilt $(2.13 \%)$, and mosaic virus $(1.23 \%)$ was observed.

Similarly, at flowering stage Fusarium wilt $(58 \%$ in Sumandri, $47 \%$ in Jaranwala, $49 \%$ in Pansera, $54.56 \%$ and $50.4 \%$ at two locations of Manawala; Alternaria (15\%),
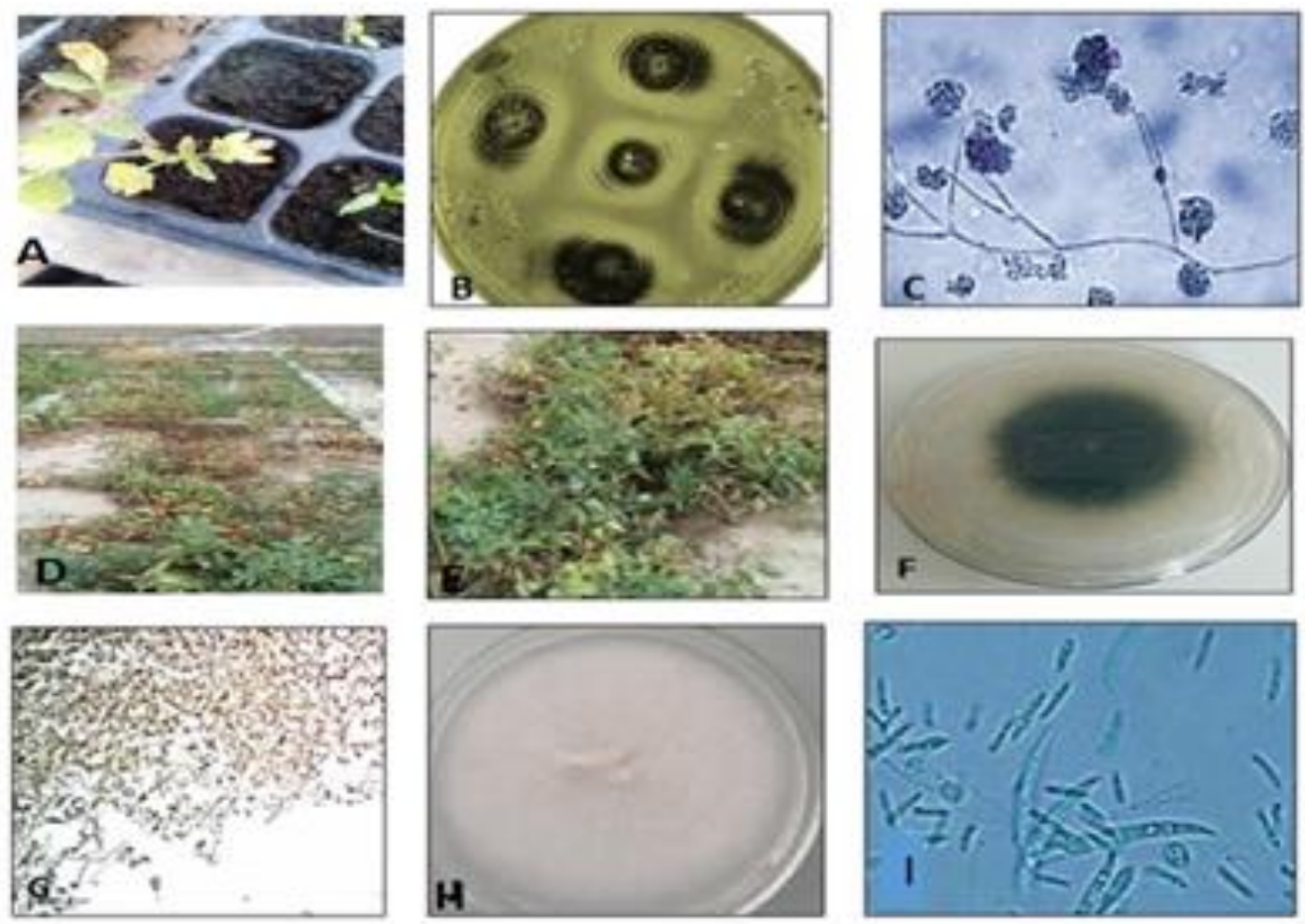

Figure 2. (A) Symptoms of Verticillium wilt, (B) Colony growth of Verticillium wilt, (C) Spore and mycelia of Verticillium wilt, (D) Symptoms of early blight, (E) Symptoms of Fusarium wilt, (F) Colony growth of Alternaria solani, (G) spores and mycelia of A. solani, (H) Colony growth of fol and ( I) Spores of Fol 
Table 1: Prevalence of tomato diseases at various growth stages from selected locations

\begin{tabular}{|c|c|c|c|c|c|c|c|}
\hline Areas & & $\begin{array}{c}\text { Fusarium } \\
\text { Wilt }\end{array}$ & $\begin{array}{l}\text { Early } \\
\text { Blight }\end{array}$ & $\begin{array}{c}\text { Damping- } \\
\text { off }\end{array}$ & $\begin{array}{c}\text { Plant Paratactic } \\
\text { Nematodes }\end{array}$ & $\begin{array}{c}\text { Verticillium } \\
\text { Wilt }\end{array}$ & TMV \\
\hline \multirow[t]{3}{*}{ Jhang } & Nursery & + & - & + & - & + & - \\
\hline & Flowering stage & ++ & + & - & - & + & - \\
\hline & Fruiting stage & + & + & - & + & + & + \\
\hline \multirow{3}{*}{ Faisalabad } & Nursery & + & - & + & - & - & - \\
\hline & Flowering stage & ++ & +- & + & - & +- & - \\
\hline & Fruiting stage & ++ & + & - & + & + & + \\
\hline \multirow[t]{3}{*}{ Manawala } & Nursery & + & - & + & - & - & - \\
\hline & Flowering stage & ++ & - & - & - & - & - \\
\hline & Fruiting stage & + & +- & - & + & + & + \\
\hline \multirow[t]{3}{*}{ Gojra } & Nursery & + & - & + & - & - & - \\
\hline & Flowering stage & ++ & - & - & - & +- & - \\
\hline & Fruiting stage & + & +- & - & + & - & ++ \\
\hline \multirow[t]{3}{*}{ Sumandri } & Nursery & + & - & + & - & - & - \\
\hline & Flowering stage & ++ & - & - & - & + & - \\
\hline & Fruiting stage & + & + & - & + & ++ & + \\
\hline \multirow[t]{3}{*}{ Toba } & Nursery & + & - & + & - & + & - \\
\hline & Flowering stage & ++ & - & - & - & ++ & - \\
\hline & Fruiting stage & + & +- & - & + & ++ & ++ \\
\hline \multirow[t]{3}{*}{ Jarawala } & Nursery & + & - & + & - & + & - \\
\hline & Flowering stage & ++ & + & - & - & ++ & - \\
\hline & Fruiting stage & + & - & - & + & + & + \\
\hline \multirow[t]{3}{*}{ Sheikhupura } & Nursery & + & +- & + & - & - & - \\
\hline & Flowering stage & ++ & - & - & - & + & - \\
\hline & Fruiting stage & + & - & - & ++ & - & ++ \\
\hline \multirow[t]{3}{*}{ Chiniot } & Nursery & + & + & + & - & + & - \\
\hline & Flowering stage & + & + & - & - & + & - \\
\hline & Fruiting stage & + & + & - & + & + & + \\
\hline \multirow{3}{*}{ Sargodha } & Nursery & + & + & + & - & + & - \\
\hline & Flowering stage & + & + & - & - & + & - \\
\hline & Fruiting stage & + & + & - & + & + & + \\
\hline Naya & Nursery & + & - & + & - & + & - \\
\hline \multirow[t]{2}{*}{ Lahore } & Flowering stage & ++ & +- & - & - & + & - \\
\hline & Fruiting stage & + & - & - & + & + & + \\
\hline \multirow[t]{3}{*}{ Pansera } & Nursery & + & - & + & - & + & - \\
\hline & Flowering stage & ++ & - & - & - & + & - \\
\hline & Fruiting stage & + & - & - & + & + & + \\
\hline \multirow{3}{*}{$\begin{array}{l}\text { Mandi Shah } \\
\text { Jewana }\end{array}$} & Nursery & + & - & + & - & + & - \\
\hline & Flowering stage & ++ & +- & - & - & + & - \\
\hline & Fruiting stage & + & + & - & + & + & + \\
\hline
\end{tabular}

$(+)$ attack, (++) severe attack, (+-) presence of disease, (-) No disease

damping off $(0.5 \%)$, nematodes $(0.5 \%)$, Verticillium wilt (32\%) and mosaic virus $(0.5 \%)$ were found. At fruiting stage Fusarium wilt (68\% in Sumandri 63\%, Jaranwala 65\%, Pansera $60 \%$ and Manawala 57\%), Alternaria (51\%), damping off $(0.5 \%)$, nematodes $(34 \%)$, Verticillium wilt $(32 \%)$ and mosaic virus $(42 \%)$ were noticed. In Toba similar incidence of tomato specific diseases were observed. In Toba Tek Singh all of diseases were observed either at nursery, flowering or fruiting stages. Incidence of Fusarium wilt was $28 \%, 25.6 \%$, and $26.7 \%$ at Gojra 1, Gojra 2 and Naya Lahore respectively while, Alternaria (3.51\%), damping off (28.76\%), nematodes (2.34\%), Verticillium wilt $(22.37 \%)$ and mosaic virus (1.23\%) were also observed. At fruiting and flowering stages Fusarium wilt was dominant as compared to other diseases.

In Sheikhupura, disease incidence like Fusarium wilt, damping off, Verticillium wilt, nematodes, and mosaic virus were present with different intensity and were recorded at three selected physiological growth stages. In nursery Fusarium wilt (26.7\%) Alternaria (5.67\%) damping off 
Table 2. Mean sum of square of disease incidence and disease severity at various stages and various locations.

\begin{tabular}{lccccccc}
\hline & $\begin{array}{c}\text { Disease } \\
\text { incidence at } \\
\text { Nursery } \\
\text { stage }\end{array}$ & $\begin{array}{c}\text { Disease } \\
\text { incidence at } \\
\text { Flowering } \\
\text { stage }\end{array}$ & $\begin{array}{c}\text { Disease } \\
\text { incidence at } \\
\text { Fruiting } \\
\text { stage }\end{array}$ & $\begin{array}{c}\text { Disease } \\
\text { severity at } \\
\text { Nursery } \\
\text { stage }\end{array}$ & $\begin{array}{c}\text { Disease } \\
\text { severity at } \\
\text { Flowering } \\
\text { stage }\end{array}$ & $\begin{array}{c}\text { Disease } \\
\text { severity at } \\
\text { Fruiting } \\
\text { stage }\end{array}$ & $\begin{array}{c}\text { Disease } \\
\text { prevalence }\end{array}$ \\
\hline Locations & $270.48^{* *}$ & $286.9^{* *}$ & $779.2^{* *}$ & $270.48^{* *}$ & $864.6^{* *}$ & $312.8^{* *}$ & $94.59^{* *}$ \\
Pathogens & $4362.24^{* *}$ & $20673.8^{* *}$ & $11209.6^{* *}$ & $4362.24^{* *}$ & $13345.2^{* *}$ & $22433.7^{* *}$ & $4656.47^{* *}$ \\
Location*Pathogens & $257.95^{* *}$ & $345.6^{* *}$ & $536.0^{* *}$ & $257.95^{* *}$ & $608.6^{* *}$ & $394.2^{* *}$ & $251.09^{* *}$ \\
Error & 1.79 & 1.2 & 1.8 & 1.63 & 1.4 & 1.2 & 1.74 \\
\hline
\end{tabular}

Where *significant, $* *$ highly significant, ns Non-significant

Table 3: Mean of disease incidence and disease severity at various locations.

\begin{tabular}{lcccccc}
\hline Locations & $\begin{array}{c}\text { Incidence Mean at } \\
\text { fruiting stage }\end{array}$ & $\begin{array}{c}\text { Incidence Mean } \\
\text { at flowering stage }\end{array}$ & $\begin{array}{c}\text { Incidence Mean } \\
\text { at nursery }\end{array}$ & $\begin{array}{c}\text { Severity at } \\
\text { nursery }\end{array}$ & Flowering & Fruiting \\
\hline Sargodha & $40.833 \mathrm{a}$ & $19.167 \mathrm{~d}$ & $17.111 \mathrm{a}$ & $19.261 \mathrm{a}$ & $44.275 \mathrm{a}$ & $22.302 \mathrm{c}$ \\
Mandi Shah Jewana & $39.000 \mathrm{~b}$ & $16.556 \mathrm{~g}$ & $17.667 \mathrm{a}$ & $19.817 \mathrm{a}$ & $42.442 \mathrm{~b}$ & $19.122 \mathrm{e}$ \\
Sumandri & $37.167 \mathrm{c}$ & $15.500 \mathrm{~h}$ & $7.944 \mathrm{~g}$ & $10.094 \mathrm{~g}$ & $40.608 \mathrm{c}$ & $17.796 \mathrm{f}$ \\
Chiniot & $36.667 \mathrm{c}$ & $21.278 \mathrm{~b}$ & $14.889 \mathrm{c}$ & $17.039 \mathrm{c}$ & $40.108 \mathrm{c}$ & $23.288 \mathrm{~b}$ \\
Toba Tak Singh & $35.111 \mathrm{~d}$ & $18.167 \mathrm{e}$ & $13.833 \mathrm{~d}$ & $15.983 \mathrm{~d}$ & $38.553 \mathrm{~d}$ & $20.446 \mathrm{~d}$ \\
Jhang & $32.333 \mathrm{e}$ & $20.167 \mathrm{c}$ & $11.778 \mathrm{e}$ & $13.928 \mathrm{e}$ & $35.775 \mathrm{e}$ & $23.537 \mathrm{~b}$ \\
Faisalabad & $31.667 \mathrm{e}$ & $19.000 \mathrm{~d}$ & $7.944 \mathrm{~g}$ & $10.094 \mathrm{~g}$ & $34.420 \mathrm{f}$ & $22.350 \mathrm{c}$ \\
Naya Lahore & $29.500 \mathrm{f}$ & $17.167 \mathrm{fg}$ & $15.833 \mathrm{~b}$ & $17.983 \mathrm{~b}$ & $32.253 \mathrm{~g}$ & $20.538 \mathrm{~d}$ \\
Manawala & $25.000 \mathrm{~g}$ & $9.944 \mathrm{j}$ & $8.222 \mathrm{~g}$ & $10.372 \mathrm{~g}$ & $28.442 \mathrm{~h}$ & $12.742 \mathrm{~h}$ \\
Gojra & $24.833 \mathrm{~g}$ & $14.000 \mathrm{i}$ & $7.944 \mathrm{~g}$ & $10.094 \mathrm{~g}$ & $27.587 \mathrm{i}$ & $16.183 \mathrm{~g}$ \\
Jarawala & $24.667 \mathrm{~g}$ & $26.778 \mathrm{a}$ & $13.833 \mathrm{~d}$ & $15.983 \mathrm{~d}$ & $27.420 \mathrm{i}$ & $29.647 \mathrm{a}$ \\
Pansera & $23.333 \mathrm{~h}$ & $15.333 \mathrm{~h}$ & $17.667 \mathrm{a}$ & $19.817 \mathrm{a}$ & $26.087 \mathrm{j}$ & $17.613 \mathrm{f}$ \\
Sheikhupura & $21.278 \mathrm{i}$ & $17.500 \mathrm{ef}$ & $10.333 \mathrm{f}$ & $12.483 \mathrm{f}$ & $23.343 \mathrm{k}$ & $18.170 \mathrm{f}$ \\
\hline
\end{tabular}

The values sharing same alphabets are non-significantly different from each other.

(25.67\%) nematodes (1.47\%) Verticillium wilt (3.47\%) and mosaic virus $(1.23 \%)$ were noticed. At flowering stage Fusarium wilt (49.3\%) Alternaria (0.5\%) damping off (0.5\%) nematodes $(0.6 \%)$ Verticillium wilt $(43.2 \%)$ and mosaic virus $(0.5 \%)$. At fruiting stage Fusarium wilt (62\%) Alternaria (34\%) damping off $(0.5 \%)$ nematodes $(47 \%)$ Verticillium wilt $(0.5 \%)$ and mosaic virus $(40 \%)$.

In Chiniot, damping off disease of tomato was more prominent in the nurseries. At flowering stage Fusarium wilt (49\%) Alternaria $(43.5 \%)$ damping off $(0.5 \%)$ nematodes $(0.5 \%)$ Verticillium wilt (38\%) and mosaic virus $(0.5 \%)$ were dominating while at fruiting stage Fusarium wilt (51.9\%) Alternaria (44\%) damping off $(0.5 \%)$ nematodes (49\%)
Verticillium wilt (44\%) and mosaic virus (42\%) were witnessed.

Specific diseases were also monitored for disease incidence at three growth stages in district Sargodha. Verticillium wilt was mainly damaging nurseries with $35.67 \%$ disease incidence followed by damping off disease. At flowering stage examination of the crop showed dominance of Fusarium wilt up to $40 \%$ disease incidence, while at fruiting stage Fusarium and Alternaria wilts were present with an incidence of $40 \%$ and $39.5 \%$ respectively.

The morphological characters of $F$. oxysporum f. sp. lycopersici were studied from 10 days old culture plates. Fol produces three kinds of spores (macroconidia, microconidia and chlamydospore). The macro-conidia were sickle shaped

Table 4. Mean of disease incidence and disease severity for various diseases.

\begin{tabular}{lcrrrrr}
\hline Diseases & $\begin{array}{c}\text { Incidence Mean } \\
\text { at fruiting stage }\end{array}$ & Flower & Nursery & $\begin{array}{c}\text { Severity at } \\
\text { nursery }\end{array}$ & Flowering & Fruiting \\
\hline Fusarium Wilt & $47.462 \mathrm{a}$ & $56.897 \mathrm{a}$ & $23.846 \mathrm{a}$ & $25.996 \mathrm{a}$ & $51.592 \mathrm{a}$ & $60.608 \mathrm{a}$ \\
Tomato Mosaic virus & $38.974 \mathrm{~b}$ & $0.000 \mathrm{e}$ & $1.282 \mathrm{e}$ & $3.432 \mathrm{e}$ & $42.787 \mathrm{~b}$ & $0.772 \mathrm{f}$ \\
Plant Parasitic nematode & $38.974 \mathrm{~b}$ & $0.000 \mathrm{e}$ & $2.564 \mathrm{~d}$ & $4.714 \mathrm{~d}$ & $43.104 \mathrm{~b}$ & $4.491 \mathrm{~d}$ \\
Verticillium wilt & $36.077 \mathrm{c}$ & $33.205 \mathrm{~b}$ & $18.564 \mathrm{~b}$ & $20.714 \mathrm{~b}$ & $39.572 \mathrm{c}$ & $37.379 \mathrm{~b}$ \\
Alternaria blight & $23.769 \mathrm{~d}$ & $13.846 \mathrm{c}$ & $6.077 \mathrm{c}$ & $8.227 \mathrm{c}$ & $26.628 \mathrm{~d}$ & $15.702 \mathrm{c}$ \\
Damping off & $0.000 \mathrm{e}$ & $2.4615 \mathrm{~d}$ & $23.821 \mathrm{a}$ & $25.971 \mathrm{a}$ & $0.000 \mathrm{e}$ & $2.771 \mathrm{e}$ \\
\hline
\end{tabular}

The values sharing same alphabets are non-significantly different from each other 
and had septations while micro-conidia were smaller and single celled. Representative pictures of spores of fusarium and alternaria along with root knot nematodes are shown (Fig. $3)$.

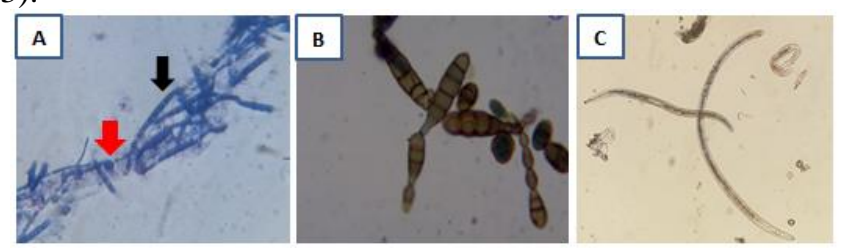

Figure 3. (A) Microconidia and macroconidia (B) Alternaria spores (C) Root Knot nematode

Epidemiological correlation with disease endemic based on months data: Results showed correlation between disease severity and environmental factors. Figure (4a) represents maximum temperature correlated with disease severity and it shows positive correlation $\left(\mathrm{R}^{2}=0.99\right)$. It also represents that disease severity increases with the rise in temperature during the survey (four months). Moreover, the pearson's correlation depicts high regression coefficient among disease severity and maximum temperature (Table 4). Minimum temperature recorded positive and significant regression coefficient with disease severity $\left(R^{2}=0.99\right)$ (Graph b). Disease severity increases with the decrease in temperature during the survey data (four months). At the same time regression table present significant relation among disease severity and minimum temperature among four months. Graph (c) present wind speed with disease severity and it shows negative regression.
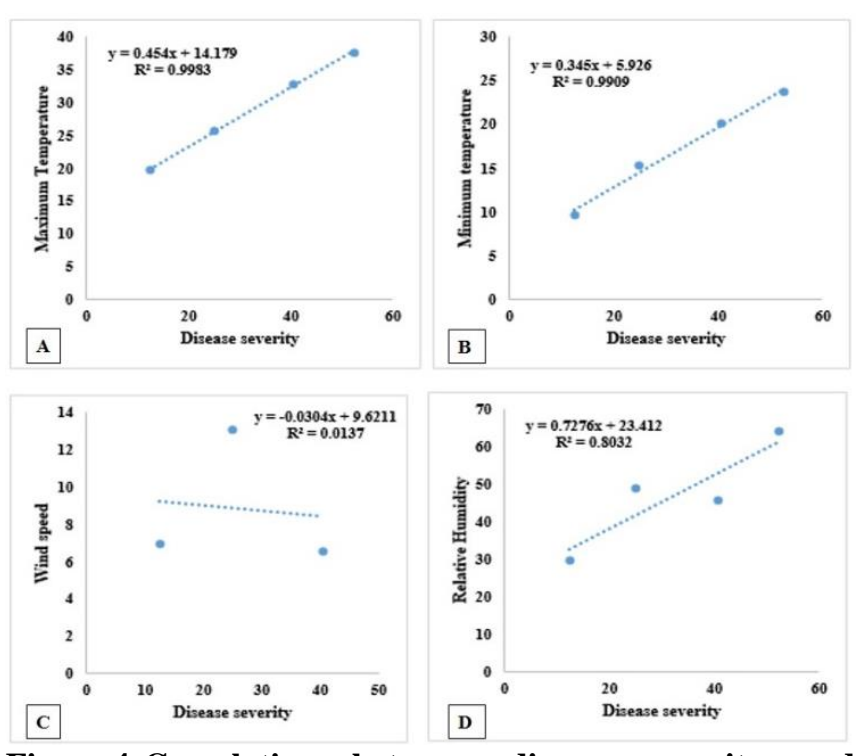

Figure 4. Correlation between disease severity and environmental factors during various months

Table 5. Correlation matrix study among maximum \& minimum temperature, relative humidity, and months.

\begin{tabular}{|c|c|c|c|c|c|}
\hline & Disease & Max. Temp & Min. Temp & Relative Humidity & Months \\
\hline \multirow[t]{2}{*}{ Max. Temp } & $0.9991 * *$ & & & & \\
\hline & 0.0009 & & & & \\
\hline \multirow[t]{2}{*}{ Min. Temp } & $0.9954 * *$ & $0.9981 * *$ & & & \\
\hline & 0.0046 & 0.0019 & & & \\
\hline \multirow[t]{2}{*}{ Relative Humidity } & $0.8962 * *$ & $0.8936 \mathrm{~ns}$ & $0.9080 \mathrm{~ns}$ & & \\
\hline & 0.0038 & 0.1064 & 0.0920 & & \\
\hline \multirow[t]{2}{*}{ Months } & $0.9986 * *$ & $0.9972 *$ & $0.9951 \mathrm{~ns}$ & $0.9178 \mathrm{~ns}$ & \\
\hline & 0.0014 & 0.0028 & 0.0049 & 0.0822 & \\
\hline \multirow[t]{2}{*}{ Wind Speed } & $-0.6720 \mathrm{~ns}$ & $0.7017 \mathrm{~ns}$ & $0.7359 \mathrm{~ns}$ & $0.6208 \mathrm{~ns}$ & $0.6667 \mathrm{~ns}$ \\
\hline & 0.3280 & 0.2983 & 0.2641 & 0.3792 & 0.3333 \\
\hline
\end{tabular}

*significant, ** highly significant, ns Non-significant (Upper value represents correlation and down value represents probability value)

Table 6. Correlation matrix study among maximum \& minimum temperature, relative humidity, and months

\begin{tabular}{lcccc}
\hline & $\begin{array}{c}\text { Disease severity of } \\
\text { different locations }\end{array}$ & $\begin{array}{l}\text { Maximum } \\
\text { Temperature }\end{array}$ & $\begin{array}{l}\text { Minimum } \\
\text { temperature }\end{array}$ & Relative humidity \\
\hline Maximum Temperature & $-0.1870 \mathrm{~ns}$ & & & \\
& 0.5408 & & & \\
Minimum Temperature & $-0.1292 \mathrm{~ns}$ & $-0.1421 \mathrm{~ns}$ & & \\
& 0.6739 & 0.6432 & $-0.3424 \mathrm{~ns}$ & \\
Relative humidity & $0.5601^{*}$ & $-0.4918 \mathrm{~ns}$ & 0.2521 & \\
& 0.0465 & 0.0878 & $0.1933 \mathrm{~ns}$ & $-0.2036 \mathrm{~ns}$ \\
Wind Speed & $-0.0859 \mathrm{~ns}$ & $0.3480 \mathrm{~ns}$ & 0.5269 & 0.5046 \\
\hline
\end{tabular}

*significant, ** highly significant, NS Non-significant (Upper value represents correlation and down value represents probability value) 
Table 7. Correlation matrix study among maximum \& minimum temperature, relative humidity, and wind speed

\begin{tabular}{lcccc}
\hline & $\begin{array}{c}\text { Disease severity of } \\
\text { different locations }\end{array}$ & $\begin{array}{c}\text { Maximum } \\
\text { Temperature }\end{array}$ & $\begin{array}{c}\text { Minimum } \\
\text { temperature }\end{array}$ & Relative humidity \\
\hline Maximum Temperature & $-0.0823 \mathrm{~ns}$ & & & \\
& 0.7892 & & & \\
Minimum Temperature & $-0.1396 \mathrm{~ns}$ & $-0.1931 \mathrm{~ns}$ & \\
& 0.6492 & 0.5273 & $-0.5523 \mathrm{~ns}$ & \\
Relative humidity & $0.4856 \mathrm{~ns}$ & $-0.3859 \mathrm{~ns}$ & 0.0503 & \\
& 0.0925 & 0.1927 & $-0.0046 \mathrm{~ns}$ & $-0.1058 \mathrm{~ns}$ \\
Wind Speed & $-0.1274 \mathrm{~ns}$ & $0.2280 \mathrm{~ns}$ & 0.9882 & 0.7309 \\
\hline
\end{tabular}

*significant, ** highly significant, ns Non-significant (Upper value represents correlation and down value represents probability value)

Table 8. Correlation matrix study among maximum \& minimum temperature, relative humidity, and locations.

\begin{tabular}{|c|c|c|c|c|c|}
\hline & $\begin{array}{l}\text { Disease severity of } \\
\text { different locations }\end{array}$ & $\begin{array}{l}\text { Maximum } \\
\text { Temperature }\end{array}$ & $\begin{array}{c}\text { Minimum } \\
\text { temperature }\end{array}$ & Relative humidity & Wind Speed \\
\hline Maximum & $-0.082 \mathrm{~ns}$ & & & & \\
\hline Temperature & 0.7892 & & & & \\
\hline Minimum & $-0.1396 n s$ & $-0.1931 \mathrm{~ns}$ & & & \\
\hline Temperature & 0.6492 & 0.5273 & & & \\
\hline \multirow[t]{2}{*}{ Relative humidity } & $0.4856 \mathrm{~ns}$ & $-0.3859 n s$ & $-0.5523 n s$ & & \\
\hline & 0.0925 & 0.1927 & 0.0503 & & \\
\hline \multirow[t]{2}{*}{ Wind Speed } & $-0.1274 \mathrm{~ns}$ & $0.2280 \mathrm{~ns}$ & $-0.0046 n s$ & $-0.1058 \mathrm{~ns}$ & \\
\hline & 0.6783 & 0.4538 & 0.9882 & 0.7309 & \\
\hline \multirow[t]{2}{*}{ Location } & $0.0071 \mathrm{~ns}$ & $-0.3580 \mathrm{~ns}$ & $0.6053^{*}$ & $-0.0852 \mathrm{~ns}$ & $-0.0190 \mathrm{~ns}$ \\
\hline & 0.9817 & 0.2297 & 0.0284 & 0.7820 & 0.9508 \\
\hline
\end{tabular}

*significant, ** highly significant, ns Non-significant (Upper value represents correlation and down value represents probability value)

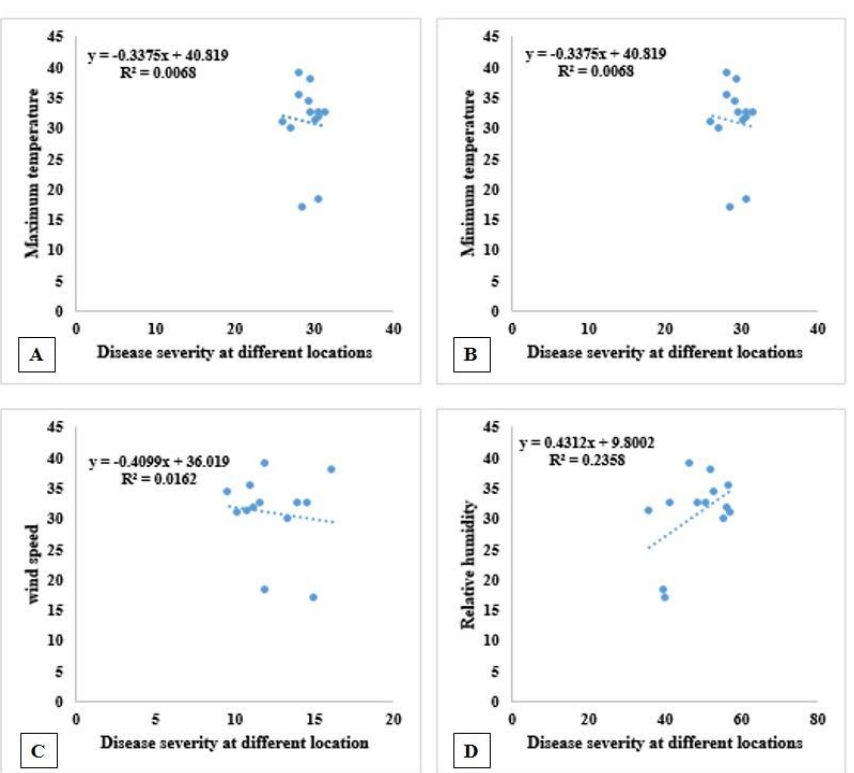

Figure 5. Correlation between disease severity and environmental factors at various locations.

Disease severity had not affected with wind speed for four months. Regression equation also presents negative effect on disease severity. Correlation table shows non-significant relation among disease severity and wind speed. Disease severity has dependency on relative humidity and positive correlation is recorded between these two variables as shown in Fig. 5. Disease severity increases with the rise in humidity for four months. Regression equation also presents relative humidity has significant effect on disease severity. At the same time correlation table present significant relation among disease severity and relative humidity among four months. Minimum and maximum temperatures have significant correlation with disease incidence.

Relative humidity and maximum temperature have significant relationship. Wind speed, maximum temperature, minimum temperature has non-significant relation at different locations.

\section{DISCUSSION}

Surveys in Punjab province show occurrence and epidemiology of various disease of tomato at different growth stages. Fusarium has been dominant at seedling stage which suggests presence of sufficient inoculum in the soil and use of seed carrying the pathogens. Lack of proper sanitation practices; use of untreated seeds and use of monoculture further promote attack of soil borne pathogens. In addition, insufficient and irrelevant use of fungicides could also enhance prevalence and incidence of fungal diseases. Suitable environmental conditions such as temperature and $\mathrm{RH}$ that are 
$>29{ }^{\circ} \mathrm{C}$ and $85 \%$ respectively accelerate infection development of Ralstonia solanacearum cells (Jonathan et al., 2014). Acidic pH (5-6) and temperatures range in 25-28 ${ }^{\circ} \mathrm{C}$ during growing season is appropriate for $\mathrm{Fol}$ disease prevalence and multiplication (Srivastava et al., 2012). Since $\mathrm{Fol}$ is soil-born as well as seed-born pathogen, seedling got infection right after emergence from infected seed. It is already reported that crop rotation decreases disease spread by discouraging the survival of soil-borne fungi (LeBlanc et al., 2017). Thus, crop rotation and introduction of resistance cultivars could lead to improved management of $\mathrm{Fol}$ wilt in tomato. Our study recorded that relatively high temperature $\left(30-38^{\circ} \mathrm{C}\right)$ and $\mathrm{RH}(30-70 \%)$ cause high incidence of necrotrophic fungi in the tomato. The similar results were reported that the environmental condition (temperature 25$28^{\circ} \mathrm{C}$ ) and soil $\mathrm{pH}(5-6)$ causes high incidence of the fungal disease (Srivastava et al., 2012). Similarly, Alvarez et al (2010) and Mrema et al. (2017) reported higher severity and disease incidence are due to soil factors, strain type, mono cropping, suitable temperature, high RH for solanacearum cell multiplication. Since, Fusarium wilt is transmitted via soil and seeds, seedlings are easily infected immediately after they emerge from infected seeds supported by external conditions. Crop rotation checks the survival of soil borne fungal diseases by minimizing soil-borne inoculum but it does not work well for pathogens having survival rate of 3-5 years in soils (Leblanc et al., 2017). The maximum disease incidence at fruiting stage was recorded at Sargodha location and minimum was recorded in Sheikhupura, while at flowering stage the maximum incidence was recorded in Jaranwala and minimum was recorded in Pansera (Table 2). Increase in the incidence of fungal diseases in these areas was supported by higher RH and maximum temperature as compared to other locations.

Conclusion: Fusarium and verticillium wilt were dominant at nursery, flowering and fruiting stages. While alternaria and damping off diseases were more common in nurseries while nematodes and mosaic viruses were commonly found during fruit setting stage. However, during fruit ripening stage plant becomes more susceptible and higher disease incidence of fungal and viral diseases were witnessed. It was also concluded that increase in maximum temperature and relative humidity favors Fusarium wilt and if such conditions sustain for longer time, it may lead to severe out-break of wilt disease in tomato.

\section{REFERENCES}

Adisa, I.O., V. L. Pullagurala, S. Rawat, J. A. HernandezViezcas, C. Dimkpa and W.H. Elmer .2018. Role of cerium compounds in Fusarium wilt suppression and growth enhancement in tomato (Solanum lycopersicum). J. Agric. Food Chem. 66:5959-5970.
Agrios, G.N. 2005. Plant pathology, 5th edn. Academic, San Diego. USA.

Akbar A, S. Hussain, K. Ullah, M. Fahim and G. S. Ali. 2018. Detection, virulence and genetic diversity of Fusarium species infecting tomato in Northern Pakistan. PLoS ONE $\quad 13$ : 0203613. https://doi.org/10.1371/journal.pone.0203613

Aloyce, A., P.A. Ndakidemi and E.R. Mbega. 2019. Survey and conventional management methods of bacterial wilt disease in open fields and greenhouses in Tanzania. J. Plan. Pathol. 101:1107-1114.

Chohan, T. Z. and S. Ahmad. 2008. An assessment of tomato production practices in Danna Katchely, Azad Jammu Kashmir. Pak J Life Soc, Sci. 6:96-102.

Di Pietro, A., M. P. Madrid, Z. Caracuel, J. Delgado-Jarana and M.I.G. Roncero. 2003. Fusarium oxysporum: exploring the molecular arsenal of a vascular wilt fungus. Molecular Plant Pathol. 4:315-25. PubMed PMID: WOS:000185632500002. pmid:20569392.

Ignjatov, M., D. Milošević, Z. Nikolić, J. GvozdanovićVarga, D. Jovičić and G. Zdjelar. 2012. Fusarium oxysporum as Causal Agent of Tomato Wilt and Fruit Rot. Pesticides Phytomedic. (Belgrade). 27: 25-31.

Jonathan, M., R. Pathak, S. Sharma and S. Sharma. 2014. Biocontrol mechanisms of Bacillus sp., isolated from tomato rhizosphere, against Fusarium oxysporum f. sp. Lycopersic." Biol. Control. 123:60-70.

LeBlanc, N., L. Kinkel and H.C. Kistler. 2017. Plant diversity and plant identity influence Fusarium communities in soil. Mycologia. 109:128-39.

Medeiros, F.C.L., M.L.V. Resende and F.H.V. Medeiros. 2009. Defense gene expression induced by a coffee-leaf extract formulation in tomato. Physiol. Mol. Plan. Pathol. 74:175-183.

Mrema, G., S. Brommonschenkel, J. Chunwongse, A. Frary, M. Ganal, R. Spivey, T. Wu, E. Earle and S. Tanksley. 2017. Map-based cloning of a protein kinase gene conferring disease resistance in tomato, Science. 262: 1432-1436

Nahar, K. and S.M. Ullah. 2012. Morphological and physiological characters of tomato (Lycopersicon esculentum Mill) cultivars under water stress. Bangl. J Agri. Res. 37:355-360.

Noonari. S., M. I. N. Memon, S. U. Solangi, M. A. Laghari, S. A. Wagan and A. A. Sethar. 2015. Economic implications of tomato production in naushahro feroze district of Sindh Pakistan. Research on Humanities and Social Sciences.5:158-70.

Rozlianah, F. and M. Sariah. 2010. Characterization of Malaysian isolates of Fusarium from tomato and pathogenicity testing. Res. J. Microbiol. 5:926-32.

Srivastava, P.K., B. D. Shenoy, M. Gupta, A. Vaish, S. Mannan and N. Singh. 2012. Stimulatory effects of 
arsenictolerant soil fungi on plant growth promotion and soil properties. Microb. Envir. 27:477-82.

Srivastava, R., A. Khalid, U. S. Singh and A. K. Sharma. 2010. Evaluation of arbuscular mycorrhizal fungus, fluorescent Pseudomonas and Trichoderma harzianum formulation against Fusarium oxysporum f. sp. lycopersici for the management of tomato wilt. Biol Cont. 53:24-31.
Wang, C., S. Zhang, R. Hou, Z. Zhao, Q. Zheng and Q. Xu. 2011. Functional analysis of the kinome of the wheat scab fungus Fusarium graminearum. PLoS Pathog.7:1002460. pmid:22216007

Zvirin, T., R. Herman and Y. Brotman. 2010. Differential colonization and defence responses of resistant and susceptible melon lines infected by Fusarium oxysporum race 1.2. Plant. Pathol.59:576-585. 